\title{
REGISTRO Y NOTAS BIONÓMICAS DE UNA NUEVA PLAGA DEL AGUACATE Laurencella colombiana (HEMIPTERA: MARGARODIDAE) EN COLOMBIA
}

\section{RECORD AND BIONOMIC NOTES OF A NEW AVOCADO PEST Laurencella colombiana (HEMIPTERA: MARGARODIDAE) IN COLOMBIA}

\author{
Zulma Nancy Gil P. ${ }^{1}$ \\ Francisco Javier Posada F. ${ }^{1}$ \\ Myriam Pérez $\mathrm{S}^{2}$ \\ Reinaldo Cárdenas M. ${ }^{3}$
}

\section{RESUMEN}

En un reconocimiento de insectos del aguacate Persea americana (Mill) en Villamaría (Caldas, Colombia), se detectó por primera vez un Hemiptera: Margarodidae. La identificación como Laurencella colombiana, la realizó el doctor Imre Foldi (Museo de Historia Natural de Paris, Francia) y la doctora Gillian W. Watson (CABI Bioscience Identification Services, Inglaterra). Este es un nuevo registro de un género y especie para Colombia y para el aguacate. El insecto es gregario, todos los estados, maduros e inmaduros, se pueden encontrar juntos. Tanto las hembras adultas, como las ninfas, se alimentan succionando savia sobre las hojas, las ramas y los troncos. El daño es severo porque causa el secamiento de los terminales y de las ramas. El insecto, se cubre con secreciones cerosas blancas y excreta abundante miel de rocío que favorece el desarrollo de fumagina.

${ }^{1}$ I.A. Disciplina de Entomología. Centro Nacional de investigaciones del Café. Cenicafé, Chinchiná, Caldas, Colombia Sede Planalto, km. 4 vía Chinchiná-Manizales. Chinchiná (Caldas) - Colombia. (E-mail: (ZNG) zulma.gil@cafedecolombia.com); (FJPF) francisco.posada@cafedecolombia.com).

2 Tecnólogo Agropecuario. Laboratorio de Producción de hongo. Disciplina de Entomología Centro Nacional de investigaciones del Café. Cenicafé, Chinchiná, Caldas, Colombia

${ }^{3}$ Investigador científico III. Disciplina de Entomología Centro Nacional de investigaciones del Café. Cenicafé, Chinchiná, Caldas, Colombia. QEPD.
Palabras clave. Reconocimiento, insecto chupador, secamiento, ramas, tronco, aguacate.

\section{SUMMARY}

In a survey carried out on avocado insect pests in Villamaria (Caldas, Colombia), for the first time a Margarodidae (Hemiptera), was found. The insect was identified by Dr. Imre Foldi (Muséum National d'Histoire Naturelle, Entomology, Paris) and Dr. Gillian W. Watson (CABI Bioscience Identification Services, England) as Laurencella colombiana. The insect lives in groups and the different stages can be found together. They feed sucking on leaves, branches and trunks. The damage is especially serious on young branches and leads to the desiccation of terminal shoots and stem. The insect covers itself by white waxy secretions and excretes abundant honeydew, which encourages the development of sooty mould.

Key words: Survey, sucking insect, desiccation, shoots, stem, avocado.

\section{INTRODUCCIÓN}

El aguacate es una fruta cuyo centro de origen es América tropical. En Colombia es una planta ampliamente cultivada en huertos o asociada a otros cultivos. En los últimos años ha recobrado importancia por la creciente demanda interna y por el potencial de exportación. En varias regiones del país, se encuentran 
extensiones apreciables en monocultivo, con objetivo comercial, ya que Colombia es uno de los países con condiciones óptimas para este árbol. Se tienen registros de excelentes producciones que hacen altamente atractivo este cultivo como negocio agrícola y se cuenta con material de propagación de variedades mejoradas y nativas seleccionadas, con recomendaciones para los diferentes pisos altitudinales, tipos de suelos y condiciones ecológicas (Salazar et al. 1978; Ríos E Tafur, 2003, Velásquez, 2006).

El manejo sanitario es uno de los aspectos más críticos de este cultivo a pesar de existir los registros y la divulgación de los insectos que se presentan como fitófagos del aguacate (Saldarriaga, 1977; Posada, 1989; Cárdenas E Posada, 2001). En Colombia, a pesar de estas publicaciones, es muy poca o nula la investigación sobre insectos plagas y sus métodos de manejo integrado. El manejo, se basa esencialmente en la aplicación calendario con regimenes de aspersión, en algunos casos semanales, de mezclas de insecticidas con fungicidas y fertilizantes, sin evaluación de la efectividad de la aplicación.

Los insecticidas que se usan son generalmente del mismo grupo químico y se aplican con énfasis en prevenir la presencia de insectos plagas. Las plantaciones de aguacate son administradas por personal sin ninguna capacitación sobre el manejo de problemas sanitarios, por lo que no están en condiciones de tomar acciones oportunas para evitar el daño o controlar apropiadamente las poblaciones de las plagas. En las visitas de campo, se tuvo la percepción que los administradores de las plantaciones no están capacitados para evaluar los problemas sanitarios ni tienen conocimiento sobre evaluación de daño, de biología, de enemigos naturales y de métodos de control de insectos plagas.

Mantener este sistema de producción del aguacate no es sostenible a largo plazo para los productores, debido a los elevados costos de producción, al alto impacto ambiental negativo generado por la contaminación de pesticidas y a los riesgos para la salud pública, ya que es un cultivo de consumo directo de la fruta y con perspectivas de exportación donde, últimamente, se exigen estándares de calidad y hay establecidos niveles máximos de residuos de plaguicidas (EC, 2004, MacLachlan, 2006).
En Colombia hay registradas alrededor de 68 especies de artrópodos que se alimentan de aguacate (Posada, 1989), pero esto no significa que todos causen daño de importancia económica. Hay especies que tienen distribución amplia y las que llegan a causar daño severo son los insectos barrenadores de tallos y de frutos: Copturomimus perseae (Hustache), Heilipus lauri Boheman (Coleoptera: Curculionidae); Stenoma catenifer Walsingham (Lepidoptera: Stenomidae) (Hoyos \& Giraldo, 1984; Cárdenas \& Posada, 2001) $\mathrm{y}$, recientemente, se registraron dos especies de trips: Frankliniella gardeniae Moulton y Selenothrips rubrocinctus Girad (Thysanoptera: Thripidae) (Echeverri E Loaiza, 2001).

Los Margarodidae son un grupo de insectos chupadores con hábitos de alimentación sobre el follaje, raíz, tallo y frutos. De esta familia hay registradas en Colombia varias especies que atacan diversas especies de cultivos y de plantas ornamentales, pero no aguacate (Posada, 1989). Atacando aguacate hay registros de Icerya purchasi Maskell en Perú y en Estados Unidos (USDA - APHIS, 2004). Esta especie, se encuentra en Colombia atacando otras plantas hospedantes, pero aun no se ha registrado sobre aguacate (Posada, 1989). Icerya aegyptiaca (Douglas) es una especie con distribución en islas del Pacifico, Asia, Australia, África y tiene estatus de especie de riesgo sanitario e invasora en Estados Unidos (USDA - APHIS, 2000). Icerya seychellarum (Westwood) e Icerya sulfurea Lindinger han sido registradas por Foldi $\varepsilon$ Watson dentro de la lista de Cocoidae que atacan aguacate a nivel mundial (Foldi $\mathcal{E}$ Watson, 2001).

En Colombia, en 1994, apareció en una plantación de aguacate en Villa Maria (Caldas, Colombia) una nueva especie de Margarodidae que se identificó inicialmente como Dosichoides sp. por la doctora Gillian W. Watson (Posada, 2000). Sin embargo, muestras enviadas más tarde al doctor Foldi en Francia, se reconoció que era una nueva especie para la ciencia y se describió como Laurencella colombiana Foldi \& Watson (Hemiptera: Margarodidae) (Foldi $\mathcal{E}$ Watson, 2001).

En la India, se presenta la especie Drosicha stebbingi (Hemiptera: Margarodidae) en el cultivo del mango. Esta especie tiene hábitos y características morfológicos similares a los de L. colombiana en aguacate. Para el control utilizan prácticas de manejo como: colocación de 
bandas engrasadas alrededor del tronco con una mezcla de aceite de ricino con ácido sulfúrico concentrado, para detener el ascenso de las ninfas que emergen de los huevos colocados por hembras adultas sobre el suelo (Williams \& Watson, 1990).

También en la India, el corte de las ramas que dan contra el suelo, se aplica como una práctica rutinaria para evitar que las ninfas asciendan a los árboles. Adicionalmente realizan la recolección de los insectos por medio de un barrido alrededor de la raíz del árbol y luego los queman en una fosa (Williams $\mathcal{E}$ Watson, 1990). Como enemigos naturales, estos autores, registraron cinco especies de Coccinelidos y Chrysopa sp., como predadores y un Ichneumonidae del género Phygadenon parasitoide de ninfas de segundo y tercer instar.

En el presente artículo, se realiza el primer registro de la especie L. colombiana en Colombia, se describen los huevos, los adultos, las hembras y los machos, destacando sus caracteres morfológicos más sobresalientes, sus hábitos y se indican algunos métodos de control que se han venido utilizando.

\section{MATERIALES Y MÉTODOS}

En un reconocimiento de insectos del aguacate realizado en la finca Guamal, municipio de Villamaría (Caldas), a $1750 \mathrm{msnm}$, con una extensión de 46 ha sembradas en aguacate y una temperatura promedio de $18^{\circ} \mathrm{C}$, se observaron poblaciones altas de un Margarodidae causando un daño severo, especialmente sobre la variedad "Winslowson", la cual se observó como la más susceptible. Muestras de los diferentes estados biológicos del insecto, se trasladaron al laboratorio de entomología de CENICAFÉ donde fueron realizadas mediciones en huevos y en adultos (machos y hembras), observaciones y descripciones sobre la morfología y la biología del insecto, toma de fotos y registro de datos.

En el campo, en la finca Guamal, también se realizaron observaciones sobre la actividad y el comportamiento del insecto. En todas las partes del árbol, se hicieron observaciones periódicas, se recogió información sobre las prácticas de manejo empleadas en la finca y se evaluó la actividad de enemigos naturales.

\section{RESULTADOS Y DISCUSIÓN}

Identificación y taxonomía. Los adultos fueron identificados por el doctor Imre Foldi del laboratorio de Entomología del Museo de Historia Natural de Paris, Francia y la doctora Gilllian W. Watson del servicio de identificación de insectos de Inglaterra, quienes encontraron que se trataba de una nueva especie y la clasificaron en el orden: Hemiptera, familia: Margarodidae, tribu: Llaveiini, especie: Laurencella colombiana Foldi $\mathcal{E}$ Watson (Foldi $\mathcal{E}$ Watson, 2001).

Nombre vulgar. En la región los agricultores la denominan "Cucaracha". Sin embargo, no existe una clara similitud con este insecto, ya que éste es blanco y posee una cobertura cerosa y sus movimientos son muy lentos. Este insecto, se observó atacando aguacate en Villamaría, Caldas, desde 1994 (Nancy Castaño, Comunicación personal).

\section{Descripción morfológica.}

Huevos. Recién puestos son de color amarillo intenso y brillantes, a medida que avanza el desarrollo embrionario, se oscurecen pasando por un color rojo intenso hasta convertirse en negro. Son de forma oval, con el corion liso y son depositados en grupo. Cada hembra tiene la capacidad de colocar de 150 - 200 huevos, los cuales miden (promedio \pm Desviación Estándar) 1,8 $\pm 0,1 \mathrm{~mm}$ de largo y $05 \pm 002 \mathrm{~mm}$ de ancho, con un tamaño de la muestra $\mathrm{N}=10$ (Figura 1.1).

Ninfas. Cuando emergen son de forma oval, de color rojo (Figura 1.2); a medida que avanzan en el desarrollo aparecen sobre el cuerpo puntos blancos debido a las secreciones cerosas que expulsan a través de los poros cerígenos. Sobre el dorso presentan tres bandas longitudinales oscuras formadas por setas negras y delgadas. Cuando pasan de un instar a otro, se cubren totalmente de secreciones. Las ninfas emergen de la exuvia a través de una abertura longitudinal en la región cefalotoráxica El orificio anal, se encuentra en la parte dorsal y en la región posterior del cuerpo y se reconoce porque a su alrededor se presenta una aureola de color blanco formada por las secreciones (Figura 1.3). Cuando se presiona el cuerpo del insecto, se puede lograr que expulse una gota de miel de rocío a través del ano. 
Adultos. La especie presenta un marcado dimorfismo sexual. Las hembras son ápteras, robustas, de color café, cubiertas por una sustancia cerosa de color blanco que es producida días antes de la oviposición (Figura 1.4). El cuerpo esta provisto de setas simples de color negro. Los segmentos, cabeza, toráx y abdomen no se diferencian fácilmente. La longitud del ápice de la cabeza al abdomen en promedio es de 11,5 $\pm 0,7 \mathrm{~mm},(n=10)$. El ancho del cuerpo es de 7,5 $\pm 0,4 \mathrm{~mm}$, $(n=10)$. La antena tiene nueve segmentos con setas largas y simples y el último segmento es más largo y delgado que los anteriores. La longitud de las antenas en promedio es de 1,3 $\pm 0,1 \mathrm{~mm},(n=10)$. Los ojos están ubicados en la base de la antena. El estilete es largo, se encuentra en la parte ventral y se extiende hasta las coxas de las patas medias.

Los machos presentan un par de alas bien formadas (Figura 1.5); el otro par está atrofiado. Las alas son anchas, más largas que el cuerpo, provistas de dos venas muy visibles y son de color gris oscuro. La envergadura alar en promedio es de 13,1 $\pm 0,4 \mathrm{~mm}$. Los ojos son prominentes y compuestos y se proyectan hacia los lados de la cabeza. El aparato bucal esta ausente. Las antenas son largas y formadas por 27 segmentos. En la mitad de cada segmento presentan numerosas setas que son largas y simples. La antena mide en promedio 5,1 $\pm 0,1 \mathrm{~mm}$. El cuerpo es de color rojo y presenta cuatro cercos largos y desiguales a ambos lados de la parte anterior del abdomen.

Hábitos y comportamiento. Cuando las ninfas emergen de los huevos colocados por las hembras en el suelo, empiezan a subir al árbol, buscan las hojas de los terminales jóvenes y se sitúan en el envés de las hojas en las nervaduras centrales y secundarias. Todos los estados son muy móviles y de hábitos gregarios, ya que por terminal se pueden encontrar numerosas colonias compuestas por individuos de diferentes instares y adultos. Si hay ramas y hojas que dan contra el suelo, se pueden encontrar poblaciones muy altas en éstas.

Las hembras adultas descienden al suelo para ovipositar (Figura 1.6); allí se recubren de secreciones blancas que protegen los huevos, los cuales no se ven a simple vista (Figura 1.7). El periodo de oviposición dura aproximadamente 15 días; cuando termina, la hembra se reduce en tamaño. La cópula se produce tanto en el suelo como en la parte aérea del árbol. Los machos son menos numerosos que las hembras y se encuentran localizados en el envés de las hojas bajeras y uno que otro en la copa del árbol.

Daño. L. colombiana es un insecto muy prolífico. Todos los instares ninfales e incluso la hembra adulta, se alimentan y se encuentran en el árbol. Al succionar la savia, se presenta un secamiento generalizado de las partes terminales del árbol (Figura 1.8). Las secreciones azucaradas de este insecto favorecen la formación de fumagina, la cual reduce la capacidad fotosintética de la planta. Además, mancha los frutos lo que incrementa los costos de postcosecha por la limpieza de la fruta. En lotes afectados de forma generalizada, se presenta fumagina en tallos, ramas, hojas, flores y frutos (Figura 1.9). El daño, se hace crítico cuando la población es alta por la reducción de la producción. El insecto, se presenta durante todos los meses pero su nivel de población puede ser variable a través del año.

El daño causado por esta especie de insecto chupador de la savia es severo y alcanza el nivel de importancia económica en la variedad "Winshowson", porque afecta en forma generalizada los árboles, se dispersa dentro del cultivo y exige la toma de medidas de control con los que se incrementan los costos de producción.

Forma de observar el insecto en el cultivo. Debido al tamaño grande, la presencia de secreciones cerosas de color blanco y el hábito de ovipositar en el suelo es fácil detectar el insecto en la planta y muestrearlo manualmente. Cuando los huevos eclosionan, se observan las ninfas por todo el tronco, subiendo a los terminales para alimentarse. También es posible observar los machos durante el período de cópula en la base del tallo y en el plato del árbol.

Enemigos naturales. Dentro de los enemigos naturales, se encontró un Coccinelidae del género Azya sp. (Figura 1.10), que se observó activo en todo el árbol y en el suelo predando sobre los huevos y las ninfas de diferentes instares. Mediante mediciones realizadas en huertos de aguacate, se pudo verificar que la población de este depredador es abundante en presencia de $L$. colombiana. Se encontraron 36 larvas del depredador en promedio por árbol con un máximo de 72 y un mínimo de 13 , mientras que la población de la plaga fue en promedio de 59 hembras con un máximo de 203 y un mínimo de cinco hembras por árbol. Estos coccinelidos 


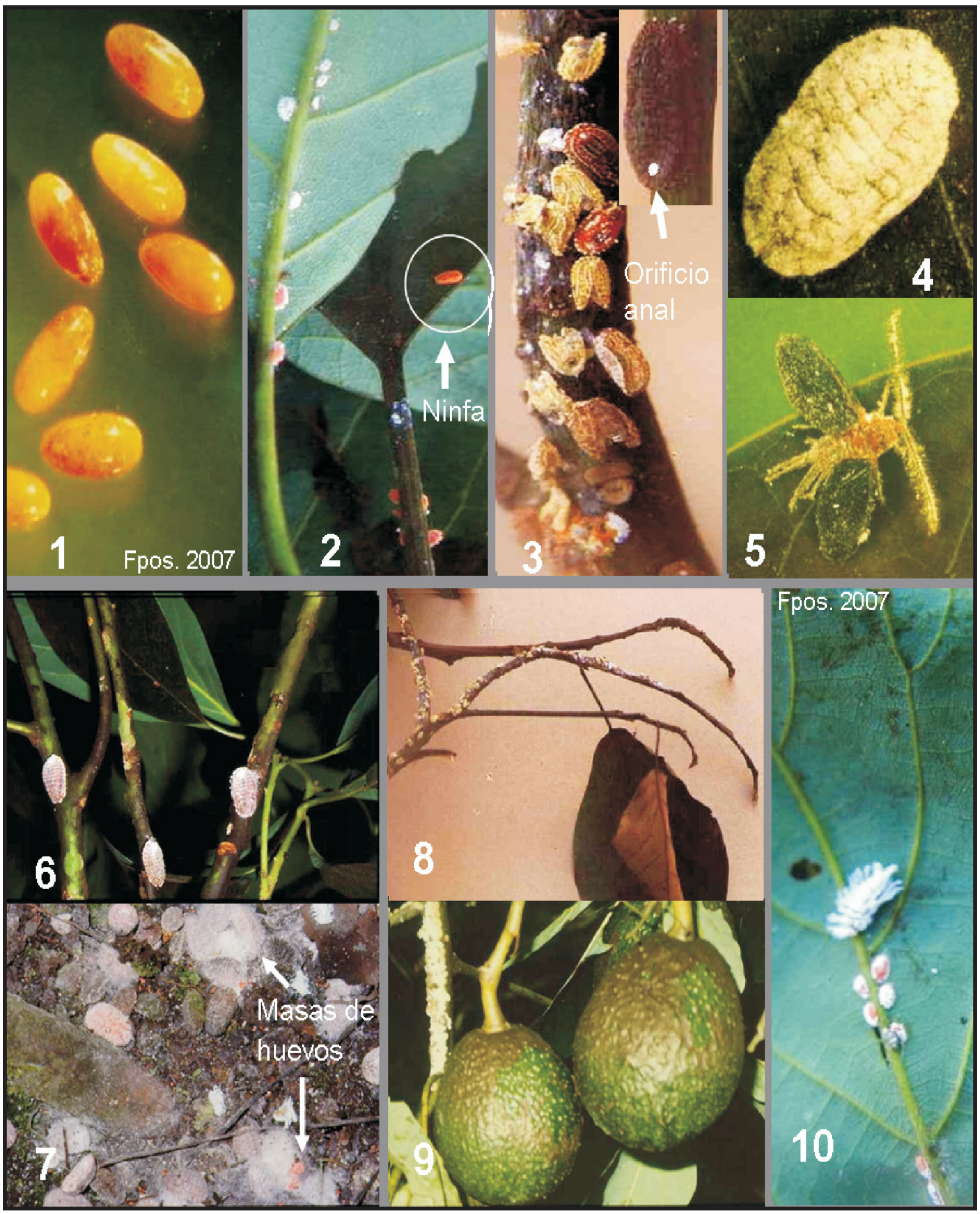

Figura 1. Estados biológicos de L. colombiana. 1. Huevos, 2. ninfas, 3. ninfas en últimos instares, 4. adulto hembra, 5. adulto macho,. 6. hembras adultas de $L$. colombiana descendiendo del árbol al suelo, 7 . masas de huevos cubiertas por secreciones cerosas, 8 . Daño de $L$. colombiana causando secamiento de las ramas, 9. fumagina en frutos, 10. larva de Azya sp. predando sobre ninfas situadas en la nervadura central de las hojas. 
empupan en las grietas de los árboles y en el suelo, lugares que es importante revisar, para protegerlos.

Medidas de manejo. Para el manejo de L. colombiana, se evaluaron varios métodos de control. Uno de ellos consistió en unas bandas plásticas amarillas colocadas en el tallo del árbol, cubiertas con una sustancia pegajosa. Estas bandas detienen el ascenso del insecto y cuando están agregados, se recolectan y se destruyen. Este método presenta limitaciones, ya que los insectos benéficos, como los coccinelidos depredadores, también quedan atrapados.

La aplicación de insecticidas ha sido la medida mayormente utilizada. Se han aplicado varios insecticidas en diferentes dosis y generalmente se aplican solos o en mezcla con emulsificantes o con aceites. Algunos de estos son: deltametrina $2,5 c c+15 c c$ de agua jabonosa / L, deltametrina $2 \mathrm{cc}+15 \mathrm{cc}$ de aceite agrícola $+20 \mathrm{cc}$ de agua jabonosa / L, chlorpyrifos $2,5 \mathrm{cc}+15 \mathrm{cc}$ de aceite agrícola $+20 \mathrm{cc}$ de agua jabonosa / L, acefato $2 \mathrm{~g} / \mathrm{L}$, acefato $2 \mathrm{~g}+20 \mathrm{cc}$ de agua jabonosa/ $\mathrm{L}$, diclorvos $2 \mathrm{cc} / \mathrm{L}$.

Las aplicaciones de insecticidas, se hacen con regímenes semanales cuando se presentan explosiones de $L$. colombiana, con el objetivo de disminuir la infestación. No hay criterios de evaluación de las aplicaciones ni de la eficacia de los productos, solo se busca encontrar el producto o mezcla que solucionen el problema temporalmente. Este enfoque de manejo, se debe superar estableciendo un programa basado en la investigación y en la evaluación de métodos de control.

Esto es urgente, ya que para productos de exportación, como el aguacate, se han establecido límites máximos de residuos de plaguicidas lo que implica que se debe vigilar la trazabilidad y la inocuidad (EC, 2004; FAO/WHO, 2006). Esto se puede constituir en una oportunidad para agregarle valor a la fruta por producción limpia y buscarle mercado con ese tipo de rotulación, ahora que existe un nicho de mercado en crecimiento con un grupo de consumidores sensible a estos tópicos y dispuestos a pagar por frutas producidas amigablemente cuidando la biodiversidad (FAO, 2001; SBA, 2006).

Teniendo en cuenta el valor de la cosecha de aguacate y la demanda nacional e internacional, el manejo de plagas en aguacate requiere de la organización gremial de los productores, de tal manera que se invierta en la producción sostenible de este renglón agrícola, enfocada al desarrollo de métodos de manejo integrado que permitan mantener este renglón productivo a largo plazo. En Colombia, se cuenta con inventarios de insectos fitófagos del aguacate (Posada, 1989) y de algunos se tiene el conocimiento de sus aspectos bionómicos (Saldarriaga, 1977; Cárdenas \& Posada, 2001). Sin embargo, se requiere hacer mayores esfuerzos en los reconocimientos de los enemigos naturales y evaluar su potencial para el desarrollo del control biológico como una alternativa de manejo al uso generalizado de productos químicos. Igualmente, se requiere desarrollar sistemas de vigilancia y evaluación y establecer niveles de daño, con el objetivo de reducir el uso de pesticidas. El uso de éstos, se debe realizar por recomendación técnica, basada en pruebas de eficacia y en la evaluación de métodos de aplicación que aseguren su máxima eficacia y el mínimo riesgo de contaminación ambiental o presencia de residuos. Las consecuencias del uso indiscriminado de los insecticidas para controlar todo insecto que se ve en las plantaciones de aguacate es posiblemente el causante de la aparición de nuevas especies que no se conocían como plagas del aguacate (Gagné et al. 2004).

El objetivo que Colombia satisfaga la demanda interna y pueda exportar aguacate solo se puede llegar a realizar si se invierte en educación y se crean programas para la capacitación de personal que maneje las plantaciones con criterios técnicos. Se requiere más apoyo a la investigación y la capacitación en programas sanitarios, tanto de enfermedades como de entomología. Esta es una oportunidad para las universidades del país de vincularse con proyectos de investigación, como soporte al desarrollo de este importante renglón agrícola. Esto es algo que se viene haciendo en California, Chile, México y Nueva Zelandia, países que han logrado convertir el desarrollo de la producción de aguacate en un renglón agrícola de gran importancia económica, basado en la investigación y en la educación (Hofshi, 2002; CAC E CMCC, 2003; CAC, 2006; Sagarpa, 2006). En California, se cuenta con programas de control biológico, manejo racional de pesticidas y monitoreo de las poblaciones de plagas, complementado con medidas de cuarentena que permiten manejar, en forma racional y a bajo costo, un reducido número de insectos plagas (Gustafson, 1979, Hoddle et al. 2002, UCDAVIS, 2007). 


\section{AGRADECIMIENTOS}

Los autores expresan sus agradecimientos al Señor Nestor Buitrago propietario de la finca el Guamal por permitir la colección de muestras de L. colombiana y realizar observaciones de campo. A Nancy Castaño por solicitar que estudiáramos este insecto que era nuevo en la región y en el cultivo del aguacate. Al doctor Imre Foldi y a la Dra G.W. Watson quienes describieron esta nueva especie para la fauna del aguacate y de Colombia.

\section{BIBLIOGRAFÍA}

CAC; CMCC. 2003. A Pest Management Strategic Plan for Avocado Production in California. 43p. Disponible desde Internet en (con acceso 06-18-2007).

CAC. 2006. Grower Best Practices Manual. 70 p. Disponible desde Internet en . (con acceso 06-18-2007).

CÁRDENAS M., R.; POSADA F., F.J. 2001. Los insectos y otros habitantes de cafetales y platanales. Armenia (Colombia), Comité Departamental de Cafeteros del Quindío-Cenicafé, 250p.

EC, 2004. MRL pesticide. Disponible desde Internet en: http://ec.europa.eu/food/plant/protection/resources/mrl_pesticide.pdf. (con acceso 06-18-2007).

ECHEVERRI F., F.; LOAIZA M., E. 2001. Primer registro de trips (Insecta: Thysanoptera) y su comportamiento asociado a cultivos de aguacate en Caldas y Risaralda. En: CONGRESO de la Sociedad Colombiana de Entomología, 28. Pereira (Colombia), Agosto 8-10, 2001. Resúmenes. Pereira (Colombia), SOCOLEN, p.28-29.

FAO, 2001. World Markets for Organic Fruit and Vegetables - Opportunities for Developing Countries in the Production and Export of Organic Horticultural Products. Disponible desde Internet en: http:// www.fao.org/DOCREP/004/Y1669E/y1669e00. htm\#Contents. (con acceso 06-18-2007).

FAO/WHO, 2006. Pesticide residues in food 2006. Joint FAO/WHO Meeting on Pesticide Residues. FAO Plant Production And Protection Paper187. 400 p. Disponible desde Internet en: http://www.
fao.org/ag/AGP/AGPP/Pesticid/JMPR/DOWNLOAD/2006_rep/report2006jmpr.pdf. (con acceso 06-18-2007).

FOLDI, I.; WATSON, G.W. 2001. A new pest scale insect on avocado trees in Colombia, Laurencella colombiana, sp. n. (Hemiptera: Coccoidea: Margarodidae). Ann. Soc. Entomol. Fr. 37(3):367-374.

GAGNE R.J.; POSADA, F.; GIL, Z.N. 2004. A new species of Bruggmanniella (Diptera: Cecicomyiidae) aborting young fruit of avocado, Persea americana (Lauraceae), in Colombia and Costa Rica. Proc. Entomol. Soc. Wash. 106 (3):547-553.

GUSTAFSON C., D. 1979. A review of pest control in avocados. California Avocado Society 1979 Yearbook 63: 58-65. 7p. Disponible desde Internet en: http://www.avocadosource.com/CAS_Yearbooks/ CAS_63_1979/CAS_1979_PG_058-065.pdf. (con acceso 0-6-18-2007).

HODDLE, M.S., MORSE, J.G., PHILLIPS, P.A., FABER, B.A., JETTER, K.M. 2002. Avocado thrips: New challenge for growers. California Agriculture, MayJune 56 (3):103-107.

HOFSHI. R. 2002. The chilean avocado industry an overview. AvoResearch. 2 (1) 1-12. Disponible desde Internet en: http://www.avocadosource.com/Journals/AvoResearch/avoresearch_02_01_2002_Hofshi_Chile.pdf. (con acceso 06-18-2007).

HOYOS, G., L.F.; GIRALDO, L.J. 1984. Reconocimiento de los insectos barrenadores del fruto del aguacate (Persea americana Mill) y evaluación económica de su daño, en huertos de los departamentos de Caldas y Risaralda. Manizales. Tesis de grado. Universidad de Caldas. Facultad de Agronomía. Manizales. 98p.

MACLACHLAN, D. 2006. Pesticide risk profile for the feeding of avocado and mango processing waste to cattle and sheep. Department Of Agriculture, Fisheries An Dforestry Canberra. Australia. 24 p. Disponible desde Internet en: http://www.safemeat.com.au/NR/rdonlyres/9ED0AAD4-E203- 
4E9B-9C20-2F806057CE78/0/avocado_mango_Jan06FINAL.pdf. (con acceso 06-18-2007).

POSADA F., F.J. 2000. Importante aclarar. Notas y Noticias Entomológicas (Socolen). 28:5-6.

POSADA O., L. 1989. Lista de insectos dañinos y otras plagas en Colombia. Bogota. Instituto Colombiano Agropecuario, Boletín Técnico No 43. 662p.

RÍOS C., D.; TAFUR R., R. 2003. Variedades de aguacate para el trópico: Caso Colombia. Proceedings V World Avocado Congress (Actas V Congreso Mundial del Aguacate). p.143-147.

SAGARPA. 2006. Campaña manejo fitosanitario del aguacatero. 3p. Disponible desde Internet en: http://www.sagarpa.gob.mx/dlg/michoacan/agricultura/sanidad/campmanejofitosanitarioaguacatero.pdf (con acceso 06-18-2007).

SALAZAR C., R.; TORRES M., R.; SERNA V., J.; GALLEGO V., M.A. 1978. Resultados preliminares en la evaluación de ocho variedades de aguacate Persea americana Mill. en zona cafetera colombiana. Revista ICA (Colombia) 13(3):425-435.

SALDARRIAGA V., A. 1977. Plagas del aguacate y su control. Curso sobre frutales. Compendio No 20. Bogotá. ICA, p.45-70.

SBA, 2006. Organic agriculture - alternative food production. 20p. Disponible desde Internet en: http://www.sba-int.ch/pdffiles/Organic.pdf. (con acceso 06-18-2007).
UCDAVIS. 2007. Avocado Pest Management. Disponible desde Internet en . (con acceso 06-18-2007).

USDA - APHIS, 2000. Regulated Plant Pest List. 11p. Disponible desde Internet en: http://www.invasive. org/regulatedpestlist.pdf. (con acceso 06-182007).

USDA - APHIS, 2004. Importation of 'Hass' Avocado (Persea americana) Fruit from Peru into the Continental United States. Raleigh, NC. 87p. Disponible desde Internet en: http://www.aphis.usda. gov/peer_review/downloads/APHIS-2006-00720002\%5B1\%5D.pdf. (con acceso 06-18-2007).

VELÁSQUEZ P., J.A. 2006. Identificación del aguacate como un rubro importante de grandes oportunidades comerciales, según los acuerdos de integración, los nuevos tratados comerciales y el comercio mundial globalizado. Secretaría de Productividad y Competitividad Gobernación de Antioquia. 10p. Disponible desde Internet en: http://www.politecnicojic.edu.co/encuentroaguacate/memorias/17/jorge_velasquez.pdf. (con acceso 06-18-2007).

WILLIAMS, D.J.; WATSON, G.W. 1990. The scale insects of the tropical south pacific region part 3: the soft scales (Coccidae) and other families. C.A.B Internal Inst. Entomol. Wallingford. p.15-18.

Recibido: mayo 16 de 2007

Aceptado: octubre 6 de 2007 